\title{
High-Resolution EUV Lithography using a Multi-Trigger Resist
}

\author{
C. Popescua,b, D. Kazazisc, A. McClelland, , G. Dawson', J. Rothe, \\ W. Theis ${ }^{a}$, Y. Ekincic ${ }^{c}$ A.P.G. Robinson ${ }^{b, d}$ \\ aSchool of Physics and Astronomy, University of Birmingham, UK. \\ bSchool of Chemical Engineering, University of Birmingham, UK. \\ cLaboratory for Micro and Nanotechnology, Paul Scherrer Institut, 5232 Villigen PSI, \\ Switzerland \\ dIrresistible Materials, Birmingham Research Park, Birmingham, UK \\ eNano-C, 33 Southwest Park, Westwood, MA, USA. \\ e-mail: a.p.g.robinson@bham.ac.uk
}

\begin{abstract}
As minimum lithographic size continues to shrink, the development of techniques and resist materials capable of high resolution, high sensitivity and low line edge roughness (LER) have become increasingly important for next-generation lithography. In this study we present results where the behaviour of the resist is driven towards the multi-trigger regime by manipulating the resist formulation. We also present results obtained after enhancements of the base molecule to give high resolution, better LER, and a significant sensitivity enhancement of $40 \%$ over the standard material. Finally, we present the inclusion of non-metallic high-Z elements into the formulation to allow for a further reduction in LER at the same resolution and sensitivity as seen for the enhanced MTR molecule, indicating a direction for further improvements.
\end{abstract}

\section{INTRODUCTION}

The newly introduced Irresistible Materials (IM) multi-trigger resist concept [1] has been designed to address the trade-off relationship between sensitivity, resolution and LER. The multi-trigger concept employed in our system enables high sensitivity but also incorporates a quenching behaviour into the chemistry to improve resolution, and reduce the materials stochastics impact of a separate quencher. The multi-trigger material consists of an MTR molecule and a crosslinker, which together represent the resist matrix, together with a photoacid generator (PAG). EUV light generates photoacids, as with a traditional chemically-amplified resist (CAR), but the response of the resist matrix implements a logictype function. Photoacids will activate matrix molecules but the reaction will only proceed where an MTR molecule and a crosslinker are simultaneously activated in close proximity to each other. In this case the matrix molecules will react together and release both acids. Where a matrix molecule is activated without an active counterpart in proximity then the acid will be retained without an exposure reaction occurring, thus removing the acid from the reaction. This behaviour allows a high sensitivity response at a certain dose threshold, but turns the resist response off much more quickly as the dose decreases, leading to sharper lines and lower LER.

Extreme Ultraviolet (EUV) Lithography IX, edited by Kenneth A. Goldberg, Proc. of SPIE Vol. 10583, 105831L · C 2018 SPIE · CCC code: 0277-786X/18/\$18 · doi: 10.1117/12.2297406 
We have previously reported [1] an improvement in sensitivity and LER using enhanced versions of our baseline material. Results of NXE exposures in this resist system have also been presented. [2] In this study we present the results of our development of the nextgeneration of MTR resists, screened at the PSI tool and the MET. We show results obtained by driving the resist behaviour towards the MTR regime, and through formulation variations, and present our strategy of work to improve the lithographic performance of our resist system.

\section{EXPERIMENTAL}

The resist samples were prepared by dissolving the individual components in ethyl lactate except for the high-Z formulations, in which case all the components were dissolved in cyclohexanone. The solutions were then combined in various weight ratios and concentrations to give a range of formulations. Some of the samples contained a nonmetallic high-Z additive in a low concentration, which was added to the resist formulation in the final solution, whilst in other cases this functionality was integrated in to the resist components.

The resist was spun onto a proprietary carbon underlayer that we have developed consisting of a mixture in equal parts of a fullerene derivative and an epoxy crosslinker, or onto a commercial underlayer, Brewer Scientific E2 STACK AL412-302.

After spin-coating of the resist the samples received a post application bake (PAB) of $60^{\circ} \mathrm{C}$ for 3 minutes except the samples containing alternative crosslinker CL2, which were baked at $90^{\circ} \mathrm{C}$ for $1 \mathrm{~min}$. EUV exposures were performed using the XIL-II interference lithography tool at the Paul Scherrer Institute, Switzerland [3] and using the MET at the Lawrence Berkeley National Laboratory, USA. After exposure the samples did not receive a post exposure bake but were developed in n-butyl acetate for 60 seconds followed by 15 seconds of MIBC rinse.

Exposed samples were analyzed with a scanning electron microscope (SEM) in top-down view. Critical dimension (CD) and LER were calculated from the SEM images with the commercial software package SuMMIT. As the direct measurement of the dose at the wafer for PSI exposures was not available in this study, the exposure was measured with respect to an internal PSI reference resist calibrated against exposures of the same resist at the Intel MET [4]. For features smaller than the capability of the calibration resist, the dose was estimated from higher pitch sizes.

The baseline for the optimization is the previously introduced xMT resist system, shown in Fig. 1, from which the MTR1 series resist was developed. The molecular resin has been modified, to increase the glass transition temperature ( $\mathrm{T}_{\mathrm{g}}$ ) (MTR2), and additionally increase crosslinkability (MTR3). Alternative crosslinker and photoacid generator molecules were also introduced in the system in order to create versions of the standard material suitable for multiple applications. Crosslinker CL2 has a higher glass transition temperature to allow for higher $\mathrm{PAB}$ temperatures, and also increased crosslinking 
capability, whilst CL3 incorporates a non-metal high-Z element for increased optical density. In the same fashion we tested PAG2, a non-antimony PAG, as an option for the standard resist to be used in tracks as a metal free material, and PAG3 for complimentary (EUV+i-line) patterning. Triphenylsulfonium tosylate, which acts as a photo-decomposable quencher in epoxy based systems, was added where indicated. [5]

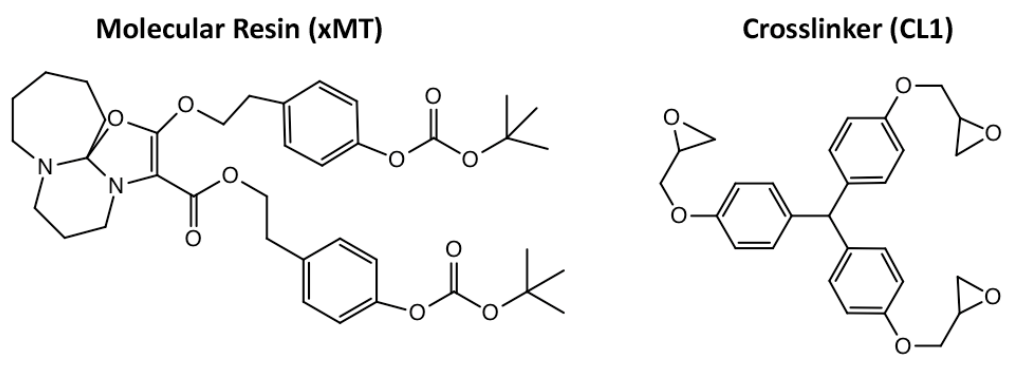

Photo-acid generator (PAG1)

Figure 1. Resist components in the baseline xMT system.

\section{RESULTS}

Below we present the results obtained by patterning the various resist formulations while investigating formulations that demonstrate simultaneous high resolution, low LER and high sensitivity. Alternative formulations were obtained by adjusting the components of the resist and their ratios, and the formulations were tested at different thicknesses. PAB and developer step optimisation were not undertaken in this work.

\subsection{Alternative formulations}

Alternative formulations were obtained by replacing the standard crosslinker in the MTR system with CL2 - a high $\mathrm{T}_{\mathrm{g}}$ crosslinker containing increased crosslinking functionality, to give MTR1400 resist. The standard MTR1200 was used as a control.

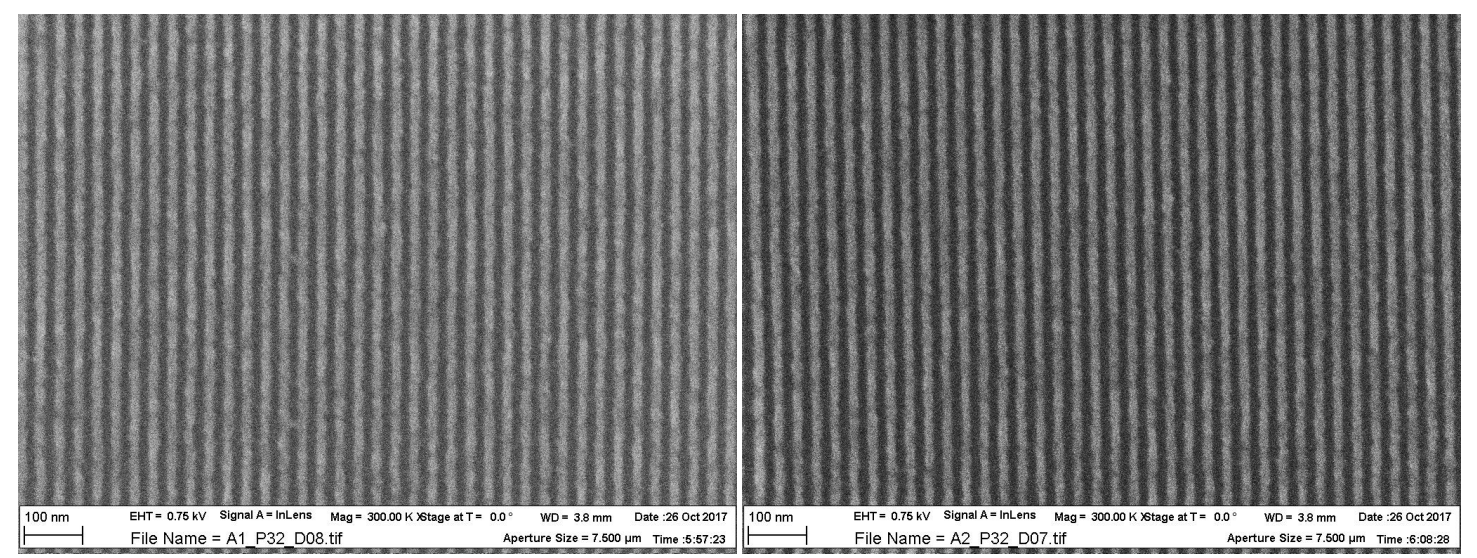

Figure 2. Top down SEM images of dense lines of $16 \mathrm{~nm}$ half-pitch (left) in standard MTR1200 formulation: 33.8mJ/cm², CD 14.8nm, LER 4.46nm; (right) MTR1400: 31.4mJ/cm², CD 15nm, LER 3.82nm 
It can be seen in Fig. 2 that a higher number of crosslinking groups in MTR1400 did not have a large impact on the dose required to pattern $16 \mathrm{~nm}$ dense lines but the LER significantly improved. This could be due to improved mechanical strength in the material caused by higher crosslinking rate, or due to the increased $\mathrm{T}_{\mathrm{g}}$.

The MTR2 formulation uses an enhanced variant of the molecular resin designed to increase $\mathrm{T}_{\mathrm{g}}$. Fig. 3(left) shows that the performance of MTR2230 is improved over the MTR1200 standard formulation (Fig. 2(left), and similar to the MTR1400 formulation. MTR2Z(1)30 contains an additive that is a non-metallic high-Z component to increase optical density but is otherwise formulated the same as MTR2230. Fig. 3 shows a comparison between the standard formulation - MTR2230 and the MTR2Z(1)30. The formulation containing the high-Z non-metallic additive doesn't demonstrate higher sensitivity but as in the previous case the LER is reduced by $0.51 \mathrm{~nm}$.

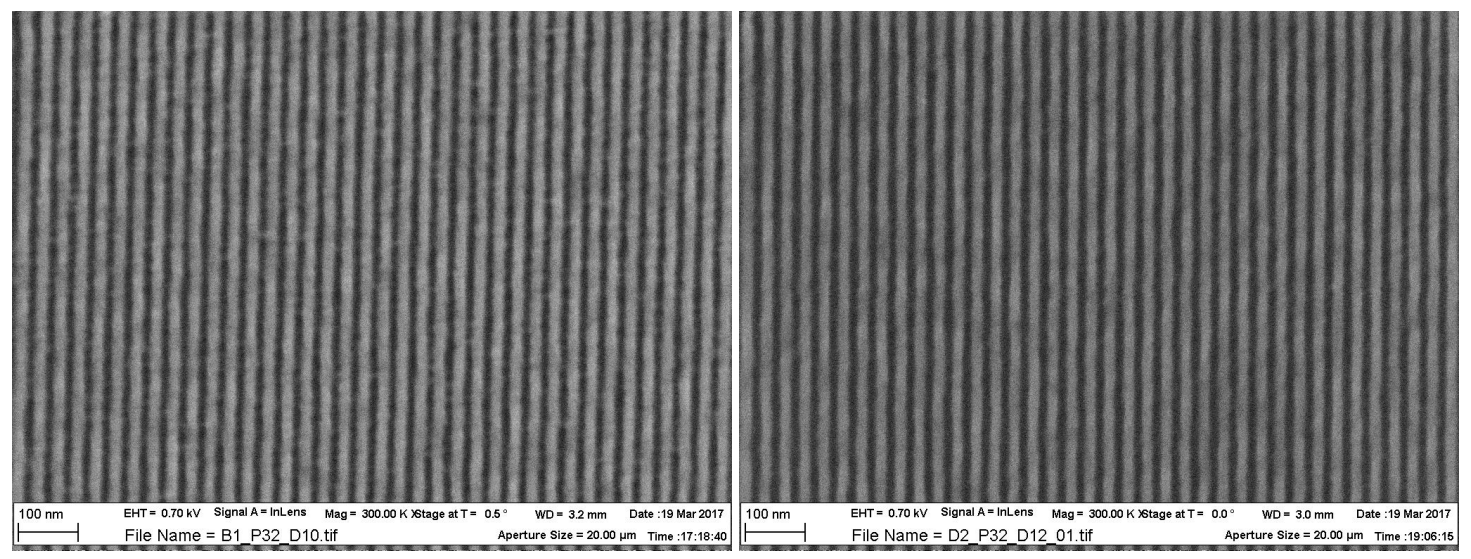

Figure 3. (left) MTR2230 $25.3 \mathrm{~mJ} / \mathrm{cm}^{2}$, CD $16.4 \mathrm{~nm}$, LER $3.28 \mathrm{~nm}$; (right) MTR2Z(1)30 with high-Z non-metallic component: $25.8 \mathrm{~mJ} / \mathrm{cm}^{2} \mathrm{CD} 15.7 \mathrm{~nm}$ LER $2.77 \mathrm{~nm}$

The results shown above led to the synthesis of a crosslinker that contains the high opacity non-metallic atom, CL3, and a new formulation, MTR2620 was created and tested at the MET in Berkeley. The patterning performance is presented in Fig. 4 and shows $13.3 \mathrm{~nm}$ and $17.5 \mathrm{~nm}$ semi-dense lines with low LER values, particularly for the $17.5 \mathrm{~nm}$ lines. The dose required to pattern these features is higher than seen using the additive, therefore further synthesis of the crosslinker will focus on increasing the sensitivity. 


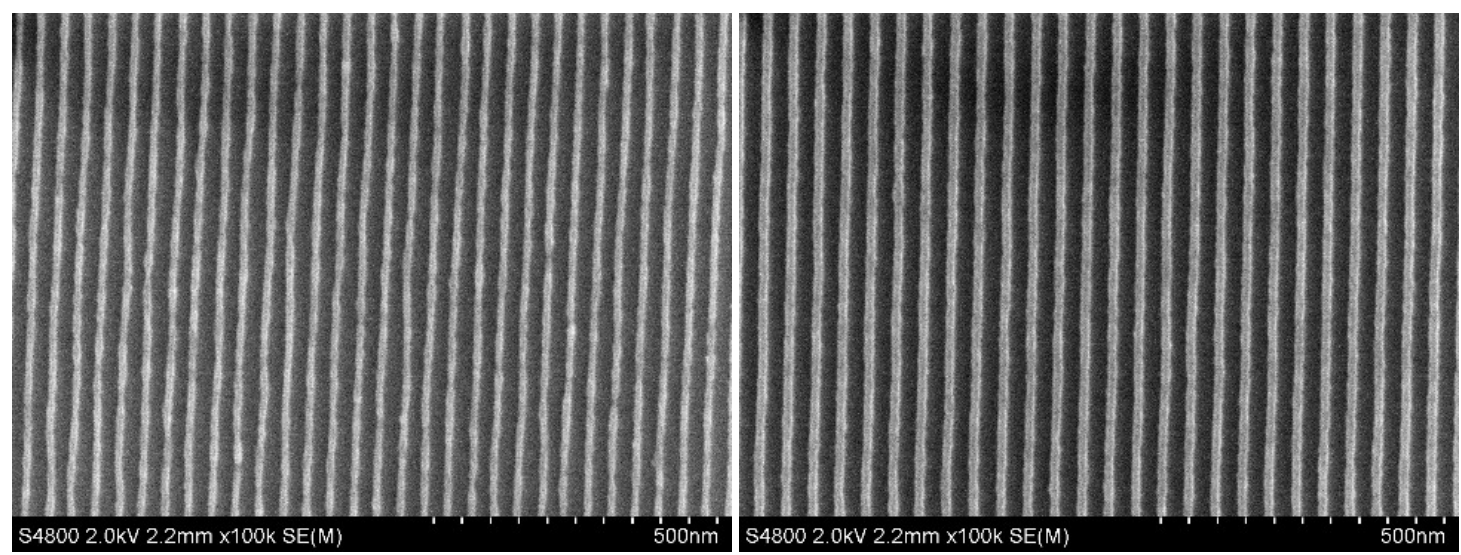

Figure 4. Top-down SEM images of MTR2620 (left) $58.8 \mathrm{~mJ} / \mathrm{cm}^{2}$, CD $13.3 \mathrm{~nm}$, LER $4.24 \mathrm{~nm}$; (right) $86.8 \mathrm{~mJ} / \mathrm{cm}^{2}$, CD $17.5 \mathrm{~nm}$, LER $2.80 \mathrm{~nm}$

MTR1300 differs from the baseline formulation in the photoacid generator molecule. The standard antimony based PAG is replaced by a non-metallic PAG. Fig. 5 shows the results obtained by patterning the two formulations in comparison.

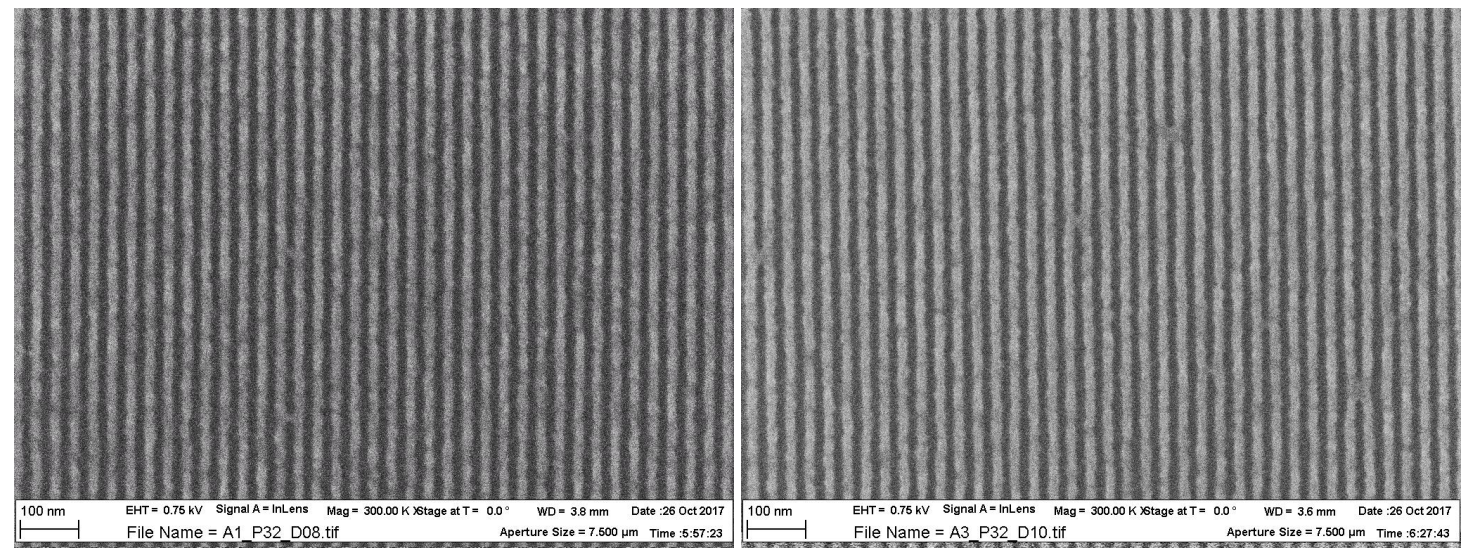

Figure 5. Top down SEM images of dense lines patterned on (left) standard MTR1200 formulation: $33.8 \mathrm{~mJ} / \mathrm{cm}^{2}$, CD $14.8 \mathrm{~nm}$, LER $4.46 \mathrm{~nm}$; (right) MTR1300: $49 \mathrm{~mJ} / \mathrm{cm}^{2}$, CD 15.6nm, LER 4.04nm

It can be seen that using the non-metallic PAG2 requires $45 \%$ more dose than the standard PAG1 but gives a smaller LER.

For MTR1Z(2)00 the photoacid generator was replaced with PAG3 - a UV sensitive molecule - and it was tested both in EUV and also i-line photolithography. Fig. 6 below shows the results. This is further evidence that MTR resist can provide a single resist 
solution to hybrid patterning approaches incorporating i-line, $193 \mathrm{~nm}$, EUV and electron beam patterning. $[6,7]$

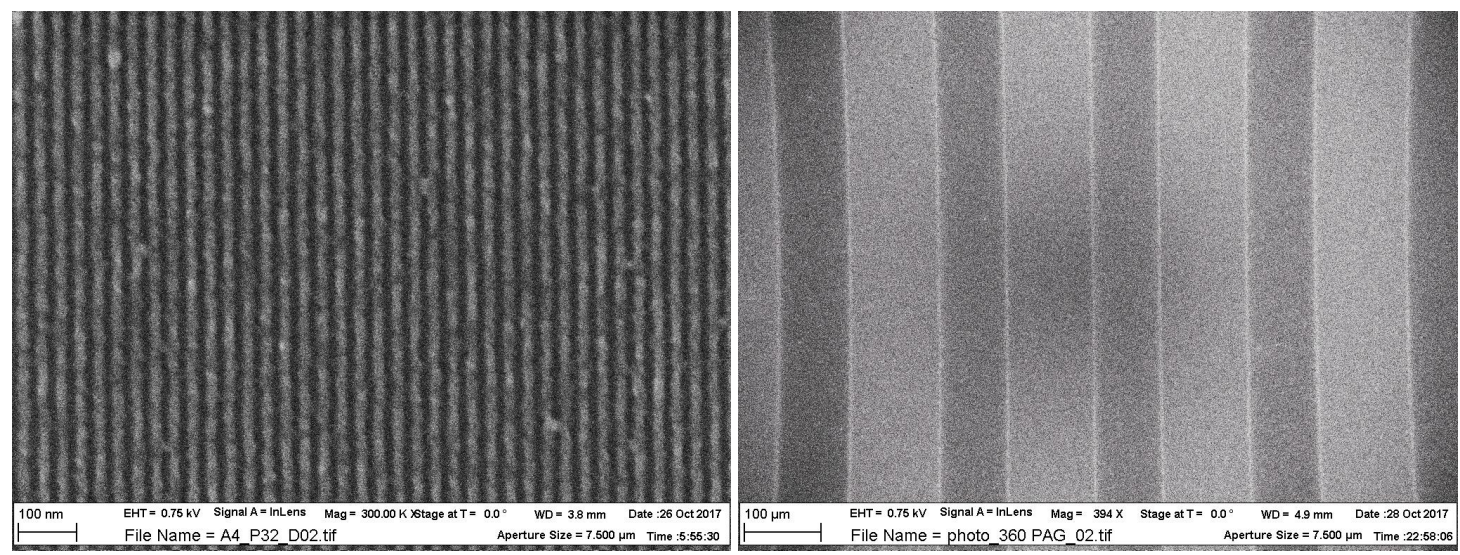

Figure 6. Top down SEM images of dense lines patterned on the MTR1Z(2)00 formulation (left) EUV-IL, $24.6 \mathrm{~mJ} / \mathrm{cm}^{2}$, CD 14.9nm, LER 6.39nm; (right) i-line photolithography

\subsection{Varying the MTR component ratio}

In order to investigate to what extent one is able to control the MTR behaviour we have varied the concentration of the MTR component in various formulations and observed the effect. Fig. 7 shows the standard MTR1210 formulation demonstrating $16 \mathrm{~nm}$ half-pitch patterns with LER of $4 \mathrm{~nm}$ at a dose of $43 \mathrm{~mJ} / \mathrm{cm}^{2}$. The effect of increasing the proportion of the MTR component is seen in the right image where LER is reduced significantly to $2 \mathrm{~nm}$ at the same pitch and similar dose.

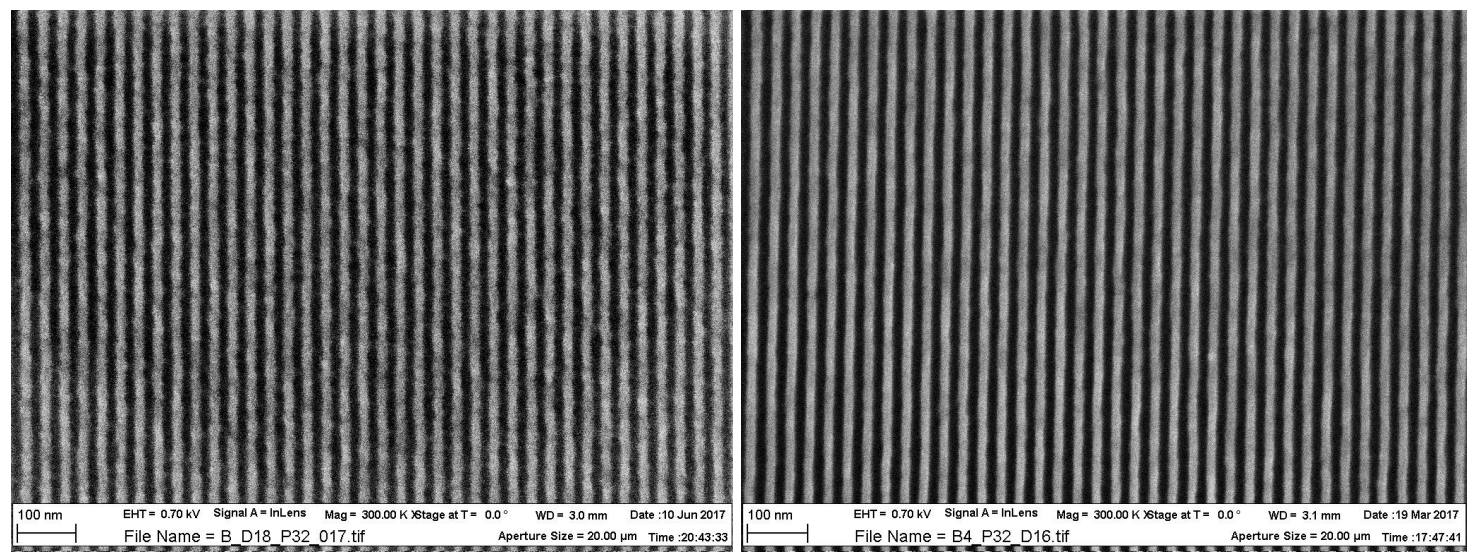

Figure 7. (left) standard MTR1210 formulation: $43.3 \mathrm{~mJ} / \mathrm{cm}^{2} \mathrm{CD} 16.2 \mathrm{~nm}$, LER $4.0 \mathrm{~nm}$, LWR $5.91 \mathrm{~nm}$; (right) high MTR2231 component formulation: $47.4 \mathrm{~mJ} / \mathrm{cm}^{2}$ CD $14.6 \mathrm{~nm}$, LER $2.06 \mathrm{~nm}$, LWR $2.52 \mathrm{~nm}$ 
A total of 4 ratios have been tested and Fig. 8 shows the trend of the dose when increasing the MTR component concentration. Table 1 presents the effect of increasing the MTR component on the LER.

\section{MTR222x, varying MTR component ratio, p32}

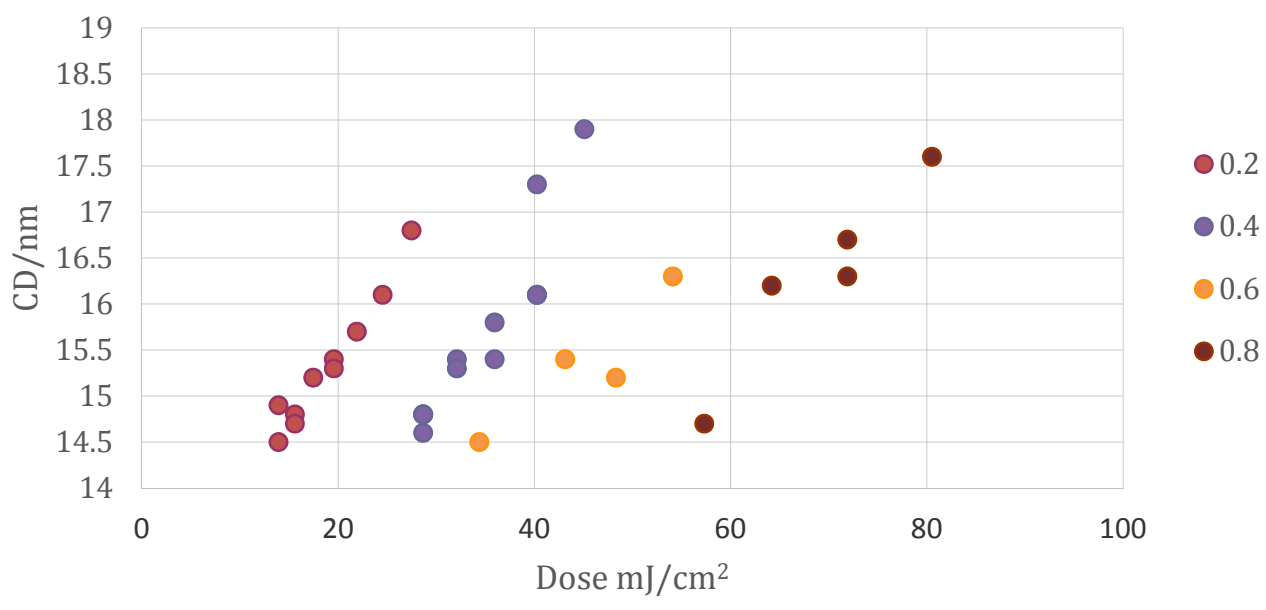

MTR322x , varying MTR component ratio, p32

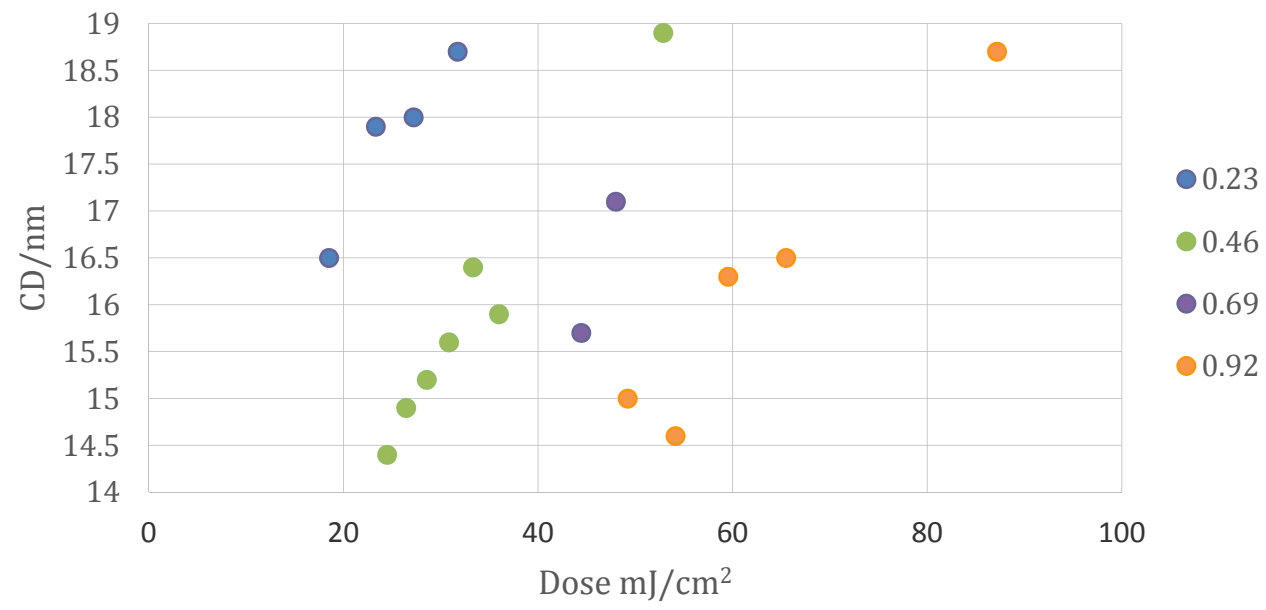

Figure 8. Dose vs CD when increasing the MTR component, in MTR222x and MTR322x resists. The MTR behaviour increases as the MTR ratio is increased from 0.2 . 


\begin{tabular}{c|c|c|c|c|c}
\hline $\begin{array}{c}\text { MTR222x } \\
\text { ratio }\end{array}$ & $\begin{array}{c}\text { LER } \\
(\mathbf{n m})\end{array}$ & $\begin{array}{c}\text { Dose } \\
\left(\mathbf{m J} / \mathbf{c m}^{2}\right)\end{array}$ & $\begin{array}{c}\text { MTR322x } \\
\text { ratio }\end{array}$ & $\begin{array}{c}\text { LER } \\
(\mathbf{n m})\end{array}$ & $\begin{array}{c}\text { Dose } \\
\left(\mathbf{m J} / \mathbf{c m}^{2}\right)\end{array}$ \\
\hline 0.2 & 6.5 & 23.3 & 0.23 & 5.9 & 13.8 \\
$\mathbf{0 . 4}$ & $\mathbf{4 . 8}$ & 36.6 & 0.46 & 4.8 & 33.9 \\
0.6 & 5.1 & 53.0 & $\mathbf{0 . 6 9}$ & $\mathbf{4 . 6}$ & 45.2 \\
0.8 & 6.1 & 66.5 & 0.92 & 4.7 & 61.0 \\
\hline
\end{tabular}

Table 1. LER and dose at 4 different MTR ratios

MTR3xxx is faster than MTR2xxx, as designed, and has a better LER with the most difference at higher MTR ratio. The best LER occurs at 0.4 ratio for MTR222x and at 0.69 for MTR322x.

\subsection{Film thickness}

In order to optimize the patterning conditions and mitigate the different failure modes in patterning such as wiggling of lines we patterned MTR2200 at various thicknesses from $18.5 \mathrm{~nm}$ to $22.5 \mathrm{~nm}$, see Fig. 9. It can be seen that a difference of $0.5 \mathrm{~nm}$ in the film thickness will impact both the dose required to pattern a given CD but also the LER.

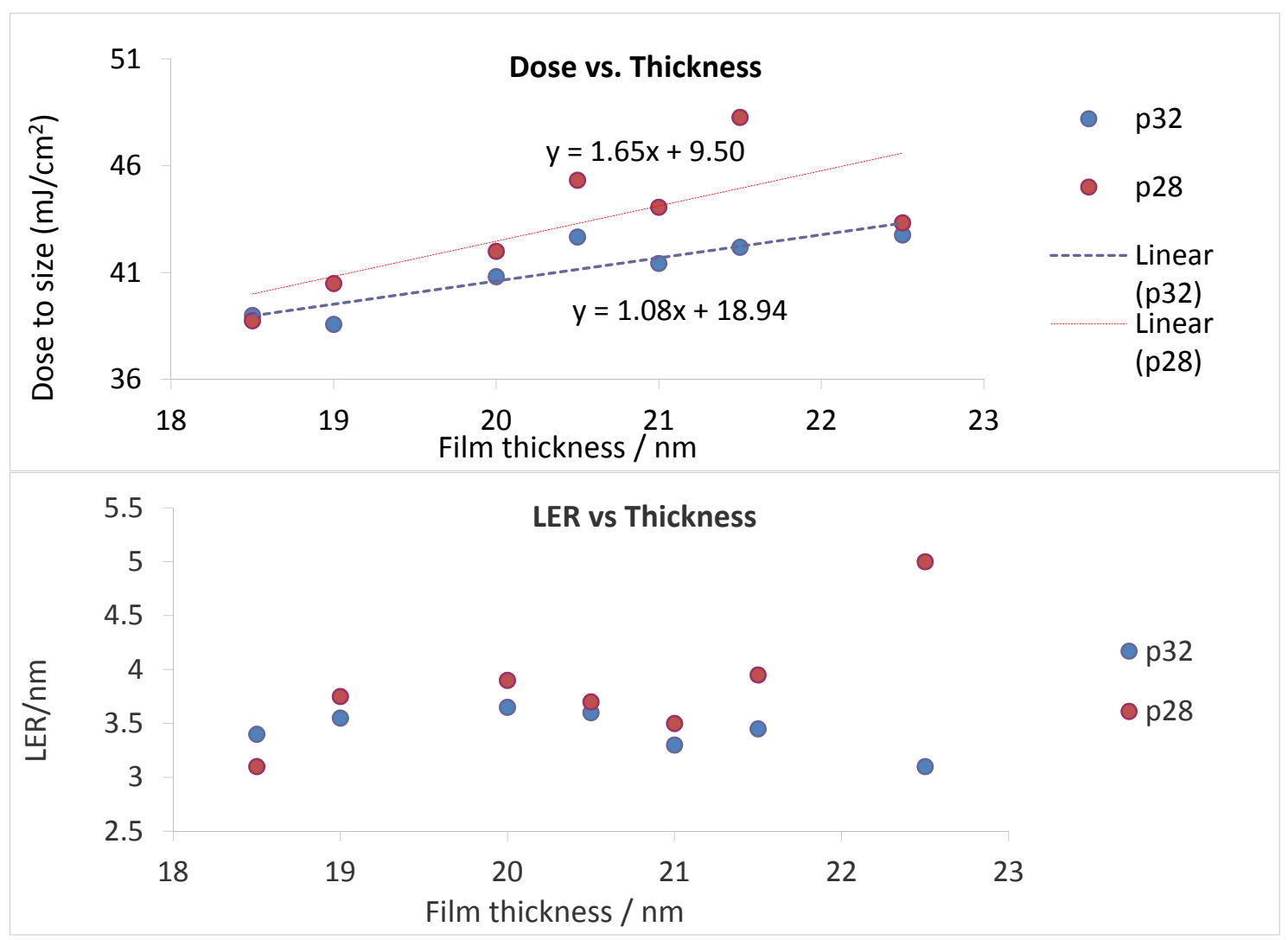

Figure 9. The effect of resist film thickness on the dose and LER 
From the results obtained in the variable thickness experiments it can be concluded that the optimum film thickness required to pattern $16 \mathrm{~nm}$ dense lines with our resist is $22.5 \mathrm{~nm}$ and $18.5 \mathrm{~nm}$ for $14 \mathrm{~nm}$ dense lines. This is clearly shown by the results in Fig. 10. This is mainly due to pattern collapse for the thicker films for the smaller features. This leads us to conclude that the effect is due to the aspect ratio of the resist. To improve the resist so that it is less prone to pattern collapse, the introduction of alternative crosslinkers such as the CL2 variant, and process changes to enhance the crosslinking of the material (whilst not increasing the dose), are being investigated.

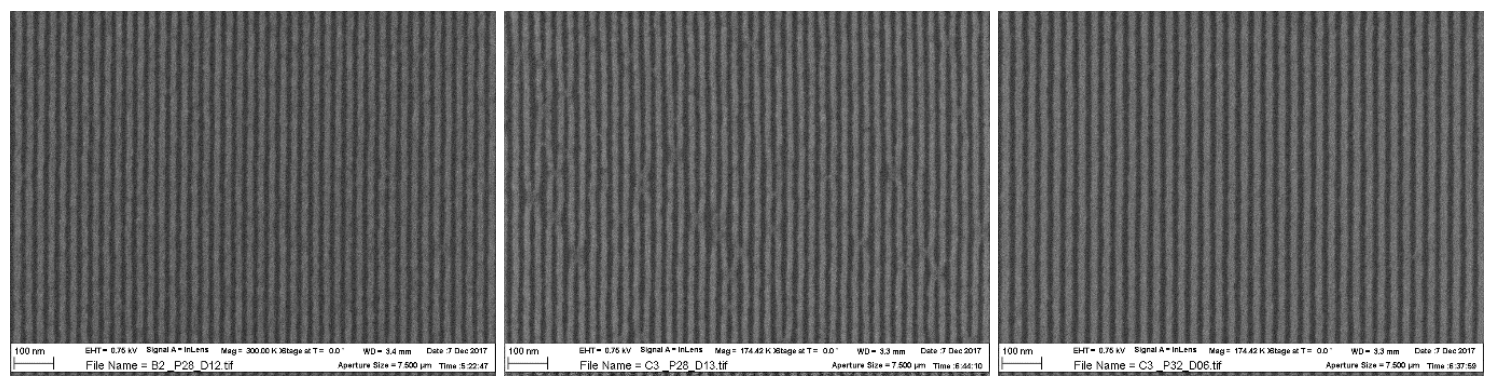

Figure 10: MTR2200 resist - (Left) 19nm film thickness (FT) at pitch 28nm with little pattern collapse, $40.8 \mathrm{~mJ} / \mathrm{cm}^{2}$, CD $14.2 \mathrm{~nm}$, LER $3.59 \mathrm{~nm}$; (middle) $22.5 \mathrm{~nm}$ FT at pitch $28 \mathrm{~nm}$ with more pattern collapse, $43.5 \mathrm{~mJ} / \mathrm{cm}^{2}, 13.6 \mathrm{~nm}, \mathrm{CD}$ $4.39 \mathrm{~nm}$, LER $4.88 \mathrm{~nm}$; (right) $22.5 \mathrm{~nm}$ film thickness at pitch $32 \mathrm{~nm}$ with little pattern collapse, $42.8 \mathrm{~mJ} / \mathrm{cm}^{2}$, CD $16.5 \mathrm{~nm}$, LER $2.92 \mathrm{~nm}$

\section{CONCLUSION}

We have shown that there are a number of materials and formulation routes to improve LER in our resist system; a high $\mathrm{T}_{\mathrm{g}}$ crosslinker reduced the LER by $0.64 \mathrm{~nm}$, a non-metallic PAG reduced the LER by $0.42 \mathrm{~nm}$. When we introduced a high-Z non-metallic additive in the system we were able to reduce the LER by $0.51 \mathrm{~nm}$ and when we used a cross-linker that contained the non-metallic high- $\mathrm{Z}$ atom the LER decreased by $1.44 \mathrm{~nm}$.

Varying the MTR component ratio in the system showed that the best LERs are achieved at 0.4 and 0.6 MTR ratios for MTR222x and MTR322x respectively. The MTR3 molecule has higher sensitivity due to the increased number of functional groups. The primary failure mode for the resist at high resolutions is through aspect ratio driven pattern collapse. Future work will focus on increasing the sensitivity of the high-Z non-metallic crosslinker, and increasing the crosslinking within the film to decrease pattern collapse. 


\section{ACKNOWLEDGEMENTS}

The authors would like to thank Ms Michaela Vockenhuber from PSI for their assistance with the EUV exposures. Part of this work was performed at Swiss Light Source (SLS), Paul Scherrer Institute, 5232 Villigen, Switzerland. The authors would like to thank the Engineering and Physical Sciences Research Council (EPSRC) for support of this project. The authors thank Irresistible Materials Ltd. and Nano-C for support and provision of resist materials. The Disco DAD 321 wafer dicer used in this research was obtained through the Birmingham Science City provided: Creating and Characterizing Next Generation Advanced Materials, with support from Advantage West Midlands (AWM) and part funded by the European Regional Development Fund (ERDF). C.P. thanks The University of Birmingham for support. This project has received funding from the EU-H2020 research and innovation program under grant agreement No 654360 having benefitted from the access provided by Paul Scherrer Institute in Villigen, Switzerland within the framework of the NFFA-Europe Transnational Access Activity.

\section{REFERENCES}

[1] Popescu C., Frommhold A., McClelland A., Roth J., Ekinci Y., Robinson A.P.G, "Sensitivity enhancement of the high-resolution xMT multi-trigger resist for EUV lithography," Proc. SPIE 10143, 101430V (2017).

[2] Vestors Y., McClelland A., Popescu C., Dawson G., Roth J., Theis W., de Simone D., Vandenberghe G., Robinson A.P.G, "Multi-trigger resist patterning with ASML NXE3300 EUV scanner," Proc. SPIE 10586, (2018).

[3] Päivänranta B., Langner A., Kirk E., David C., Ekinci Y., "Sub-10 nm patterning using EUV interference lithography," Nanotechnology 22, 375302 (2011)

[4] Ekinci Y., Vockenhuber M., Terhalle B., Hojeij M., Wang L., Younkin T. R., "Evaluation of resist performance with EUV interference lithography for sub-22 nm patterning," Proc. SPIE 8322, 83220W (2012).

[5] Lawson R.A., Frommhold A., Yang D.X., Robinson A.P.G, "Negative-tone organic molecular resists," in Robinson A.P.G., Lawson R.A. editors. Materials and Processes for Next Generation Lithography. Oxford, Elsevier (2016).

[6] Montgomery, W., McClelland A., Ure D., Roth J., Robinson A., "Irresistible Materials multi-trigger resist: the journey towards high volume readiness," SPIE 10143, 1014328 (2017).

[7] Popescu C., McClelland A., Dawson G., Roth J., Kazazis D., Ekinci Y., Theis W., Robinson A.P.G., "Multi-trigger resist for electron beam lithography," Proc SPIE 10446, 1044608 (2017). 